\title{
Holonomy groups of pseudo-quaternionic-Kählerian manifolds of non-zero scalar curvature
}

\author{
Natalia I. Bezvitnaya
}

Dedicated to Dmitri Vladimirovich Alekseevsky at the occasion of his 70th birthday

\begin{abstract}
The holonomy group $G$ of a pseudo-quaternionic-Kählerian manifold of signature $(4 r, 4 s)$ with non-zero scalar curvature is contained in $\operatorname{Sp}(1) \cdot \operatorname{Sp}(r, s)$ and it contains $\operatorname{Sp}(1)$. It is proved that either $G$ is irreducible, or $s=r$ and $G$ preserves an isotropic subspace of dimension $4 r$, in the last case, there are only two possibilities for the connected component of the identity of such $G$. This gives the classification of possible connected holonomy groups of pseudo-quaternionic-Kählerian manifolds of non-zero scalar curvature.
\end{abstract}

Keywords Pseudo-quaternionic-Kählerian manifold · non-zero scalar curvature · holonomy group $\cdot$ holonomy algebra $\cdot$ curvature tensor $\cdot$ symmetric space

Mathematics Subject Classification (2000) 53C29 · 53C26

\section{Introduction and the results}

Quaternionic-Kählerian geometry is of increasing interest both in mathematics and mathematical physics, see e.g. 1, 9, 10, 12. A pseudo-quaternionic-Kählerian manifold is a pseudo-Riemannian manifold $(M, g)$ of signature $(4 r, 4 s), r+s>1$ together with a parallel quaternionic structure $Q \subset \mathfrak{s o}(T M)$, i.e. three-dimensional linear Lie algebra $Q$ with a basis $I_{1}, I_{2}, I_{3}$ which satisfies the relations $I_{1}^{2}=I_{2}^{2}=I_{3}^{2}=-\mathrm{id}, I_{3}=I_{1} I_{2}=$ $-I_{2} I_{1}$. The holonomy group $G$ of such manifold is contained in $\operatorname{Sp}(1) \cdot \operatorname{Sp}(r, s)=$ $\operatorname{Sp}(1) \times \operatorname{Sp}(r, s) / \mathbb{Z}_{2}$. Conversely, any pseudo-Riemannian manifold with such holonomy group is pseudo-quaternionic-Kählerian. Denote by $G^{0}$ the restricted holonomy group of $(M, g)$, i.e. the connected component of identity of $G$.

The classification of connected holonomy groups of Riemannian manifolds is well known and it has a lot of applications both in geometry and physics, see e.g. 2 2,3,5,8, The corresponding problem for pseudo-Riemannian manifolds of arbitrary signature is

N.I. Bezvitnaya

Department of Mathematics and Statistics, Faculty of Science, Masaryk University in Brno, Kotlářská 2, 61137 Brno, Czech Republic.

E-mail: bezvitnaya@math.muni.cz 
solved only in some partial cases, see the recent reviews [6,7]. The difficulty appears if the holonomy group preserves a degenerate subspace of the tangent space. Here we show that the holonomy group $G \subset \operatorname{Sp}(1) \cdot \operatorname{Sp}(r, s)$ of a pseudo-quaternionic-Kählerian manifold with non-zero scalar curvature is irreducible if $s \neq r$. If $s=r$, then $G$ may preserve a degenerate subspace of the tangent space, in this case there are only two possibilities for $G$.

In [1] it is proved that the curvature tensor $R$ of a pseudo-quaternionic-Kählerian manifold of signature $(4 r, 4 s)$ can be written as

$$
R(X, Y)=\nu R_{0}+\mathcal{W}
$$

where $\nu=\frac{\text { scal }}{4 m(m+2)}(m=4 r+4 s)$ is the reduced scalar curvature,

$$
R_{0}(X, Y)=\frac{1}{2} \sum_{\alpha=1}^{3} g\left(X, I_{\alpha} Y\right) I_{\alpha}+\frac{1}{4}\left(X \wedge Y+\sum_{\alpha=1}^{3} I_{\alpha} X \wedge I_{\alpha} Y\right),
$$

$X, Y \in T M$, is the curvature tensor of the quaternionic projective space $\mathbb{P} \mathbb{H}^{r, s}$, and $\mathcal{W}$ is an algebraic curvature tensor with zero Ricci tensor. It is proved that scal $\neq 0$ if and only if the holonomy group $G$ contains $\operatorname{Sp}(1)$. In 1 it is proved also that any pseudo-quaternionic-Kählerian manifold with non-zero scalar curvature is locally indecomposable, i.e. it is not locally a product of two pseudo-Riemannian manifolds of positive dimension, or, equivalently, $G^{0}$ does not preserve any proper non-degenerate vector subspace of the tangent space.

The tangent space to the manifold $(M, g)$ can be identified with the pseudoEuclidean space $\mathbb{R}^{4 r, 4 s}$ endowed with the pseudo-Euclidean metric $\eta$ and an $\eta$-orthogonal quaternionic structure $I_{1}, I_{2}, I_{3}$, or with the pseudo-quaternionic-Hermitian space $\mathbb{H}^{r, s}$ endowed with a pseudo-quaternionic-Hermitian metric $g$. Let $s=r$ and $W \subset \mathbb{H}^{r, r}$ be an isotropic subspace of quaternionic dimension $r$. Let $p_{1}, \ldots, p_{r}, q_{1}, \ldots, q_{r}$ be a basis of $\mathbb{H}^{r, r}$ such that $p_{1}, \ldots, p_{r}$ is a basis of $W$ and the only non-zero values of the pseudo-quaternionic-Hermitian form $g$ on $\mathbb{H}^{r, r}$ are $g\left(p_{i}, q_{i}\right)=g\left(q_{i}, p_{i}\right)=1$. The maximal subalgebra $\mathfrak{s p}(r, r)_{W} \subset \mathfrak{s p}(r, r)$ preserving $W$ can be identified with the following matrix Lie algebra:

$$
\mathfrak{s p}(r, r)_{W}=\left\{\left(\begin{array}{cc}
C & B \\
0 & -\bar{C}^{t}
\end{array}\right) \mid C, B \in \operatorname{Mat}(r, \mathbb{H}), \bar{B}^{t}=-B\right\},
$$

where $\operatorname{Mat}(r, \mathbb{H})$ denotes the space of $r \times r$ quaternionic matrices. Note the following. Let $\mathbb{H}^{m}$ be an m-dimensional quaternionic vector space and $e_{1}, \ldots, e_{m}$ a basis of $\mathbb{H}^{m}$. We identify an element $X \in \mathbb{H}^{m}$ with the column $\left(X_{t}\right)$ of the left coordinates of $X$ with respect to this basis, $X=\sum_{t=1}^{m} X_{t} e_{t}$. Let $f: \mathbb{H}^{m} \rightarrow \mathbb{H}^{m}$ be an $\mathbb{H}$-linear map. Define the matrix Mat $f$ of $f$ by the relation $f e_{l}=\sum_{t=1}^{m}\left(\operatorname{Mat}_{f}\right)_{t l} e_{t}$. Now if $X \in \mathbb{H}^{m}$, then $f X=\left(X^{t} \mathrm{Mat}_{f}^{t}\right)^{t}$ and because of the non-commutativity of the quaternionic numbers this is not the same as $\operatorname{Mat}_{f} X$. Conversely, any $m \times m$ quaternionic matrix defines an $\mathbb{H}$-linear map $f: \mathbb{H}^{m} \rightarrow \mathbb{H}^{m}$. Denote by $\operatorname{Sp}(r, r)_{W} \subset \operatorname{Sp}(r, r)$ the maximal connected Lie subgroup preserving $W$, i.e. the connected Lie subgroup corresponding to the subalgebra $\mathfrak{s p}(r, r)_{W} \subset \mathfrak{s p}(r, r)$. Define the Lie subalgebra

$$
\mathfrak{h}_{0}=\mathfrak{s p}(1) \oplus\left\{\left(\begin{array}{cc}
C & 0 \\
0 & -\bar{C}^{t}
\end{array}\right) \mid C \in \operatorname{Mat}(r, \mathbb{H})\right\} \subset \mathfrak{s p}(1) \oplus \mathfrak{s p}(r, r)_{W}
$$

and denote by $H_{0}$ the corresponding connected Lie subgroup of $\operatorname{Sp}(1) \cdot \operatorname{Sp}(r, r)_{W}$.

We prove the following two statements. 
Theorem 1 Let $(M, g)$ be a pseudo-quaternionic-Kählerian manifold of non-zero scalar curvature and of signature $(4 r, 4 s)$. If its restricted holonomy group $G^{0}$ is not irreducible, then $s=r, G^{0}$ preserves an isotropic quaternionic subspace $W \subset \mathbb{H}^{r, r}$ of quaternionic dimension $r$ and either $G^{0}=H_{0}$, or $G^{0}=\operatorname{Sp}(1) \cdot \operatorname{Sp}(r, r)_{W}$.

Proposition 1 Any pseudo-quaternionic-Kählerian manifold with the restricted holonomy group $H_{0}$ is locally symmetric, i.e. its curvature tensor is parallel.

Note that if the manifold $(M, g)$ is not locally symmetric and its restricted holonomy group $G^{0}$ is irreducible, then $G^{0}=G=\operatorname{Sp}(1) \cdot \operatorname{Sp}(r, s)$, e.g. [5].

Simply connected symmetric pseudo-quaternionic-Kählerian manifolds are classified in 1]. Each such space $(M, g)$ may be represented as $M=F / K$, where $F$ is the connected group generated by transvections and $K$ is the stabilizer of a fixed point $o \in M$. The holonomy group of $(M, g)$ coincides with the isotropy representation of $K$. These spaces are exhausted by

$\begin{array}{lllll}\frac{\mathrm{SU}(p+2, q)}{S(\mathrm{U}(2) \times \mathrm{U}(p, q))}, & \frac{\mathrm{SL}(r+1, \mathbb{H})}{\mathrm{S}(\mathrm{GL}(1, \mathbb{H}) \times \mathrm{GL}(r, \mathbb{H}))}, & \frac{\mathrm{SO}_{0}(p+4, q)}{\mathrm{SO}(4) \times \mathrm{SO}_{0}(p, q)}, & \frac{\mathrm{SO}^{*}(2 l+4)}{\mathrm{SO}^{*}(4) \times \mathrm{SO}^{*}(2 l)}, & \frac{\mathrm{Sp}(p+1, q)}{\mathrm{Sp}(1) \times \mathrm{Sp}(p, q)}, \\ \frac{E_{6(-78)}}{\mathrm{SU}(2) \mathrm{SU}(6)}, & \frac{E_{6(2)}}{\mathrm{SU}(2) \mathrm{SU}(6)}, & \frac{E_{6(2)}}{\mathrm{SU}(2) \mathrm{SU}(2,4)}, & \frac{E_{6(-14)}}{\mathrm{SU}(2) \mathrm{SU}(2,4)}, & \frac{E_{6(6)}}{\mathrm{Sp}(1) \mathrm{SL}(3, \mathbb{H})}, \\ \frac{E_{6(-26)}}{\mathrm{Sp}(1) \mathrm{SL}(3, \mathbb{H})}, & \frac{E_{7(-133)}}{\mathrm{SU}(2) \mathrm{Spin}(12)}, & \frac{E_{7(-5)}}{\mathrm{SU}(2) \mathrm{Spin}(12)}, & \frac{E_{7(-5)}}{\mathrm{SU}(2) \mathrm{Spin}^{0}(4,8)}, & \frac{E_{7(7)}}{\mathrm{SU}(2) \mathrm{SO}^{*}(12)}, \\ \frac{E_{7(-25)}}{\mathrm{SU}(2) \mathrm{SO}^{*}(12)}, & \frac{E_{8(-248)}}{\mathrm{SU}(2) E_{7(133)}}, & \frac{E_{8(-24)}}{\mathrm{SU}(2) E_{7(133)}}, & \frac{E_{8(-24)}}{\mathrm{SU}(2) E_{7(-5)}}, & \frac{E_{8(8)}}{\mathrm{SU}(2) E_{7(-5)}}, \\ \frac{F_{4(-52)}}{\mathrm{Sp}(1) \mathrm{Sp}(3)}, & \frac{F_{4(4)}}{\mathrm{Sp}(1) \mathrm{Sp}(3)}, & \frac{F_{4(4)}}{\mathrm{Sp}(1) \mathrm{Sp}(1,2)}, & \frac{F_{4(-20)}}{\mathrm{Sp}(2) \mathrm{Sp}(1,2)}, & \frac{G_{2(-14)}}{\mathrm{SO}(4)}, \frac{G_{2(2)}}{\mathrm{SO}(4)} .\end{array}$

Note that the subgroup $H_{0} \subset \operatorname{Sp}(1) \cdot \operatorname{Sp}(r, r)_{W}$ is the holonomy group of the symmetric space $\frac{\mathrm{SL}(r+1, \mathbb{H})}{S(\mathrm{GL}(1, \mathbb{H}) \times \mathrm{GL}(r, \mathbb{H}))}$. This shows that the holonomy groups of other symmetric spaces are irreducible.

We get the following corollaries.

Corollary 1 Let $(M, g)$ be a pseudo-quaternionic-Kählerian manifold of non-zero scalar curvature and of signature $(4 r, 4 s)$. Then either its restricted holonomy group coincides with $\operatorname{Sp}(1) \cdot \operatorname{Sp}(r, s)$ or with $\mathrm{Sp}(1) \cdot \operatorname{Sp}(r, r)_{W}$, or $(M, g)$ is locally symmetric.

Corollary 2 Let $(M, g)$ be a complete pseudo-quaternionic-Kählerian manifold of nonzero scalar curvature and of signature $(4 r, 4 s)$. Then either its restricted holonomy group coincides with $\mathrm{Sp}(1) \cdot \mathrm{Sp}(r, s)$ or with $\mathrm{Sp}(1) \cdot \mathrm{Sp}(r, r)_{W}$, or $(M, g)$ is the factor space of a symmetric space obtained in [1] by a freely acting discrete group $\Gamma$.

Corollary 3 Let $(M, g)$ be a simply connected complete pseudo-quaternionic-Kählerian manifold of non-zero scalar curvature and of signature $(4 r, 4 s)$. If the holonomy group $G$ of $(M, g)$ is irreducible, then either $G=\operatorname{Sp}(1) \cdot \operatorname{Sp}(r, s)$, or $(M, g)$ is a symmetric space obtained in [1] different from $\frac{\mathrm{SL}(r+1, \mathbb{H})}{S(\mathrm{GL}(1, \mathbb{H}) \times \mathrm{GL}(r, \mathbb{H}))}$. If $G$ is not irreducible, then $s=r$, and either $G=\operatorname{Sp}(1) \cdot \operatorname{Sp}(r, r)_{W}$, or $(M, g)$ is isometric to the symmetric space $\operatorname{SL}(r+1, \mathbb{H})$ $\overline{S(\mathrm{GL}(1, \mathbb{H}) \times \mathrm{GL}(r, \mathbb{H}))}$.

Theorem 1 gives only the list of possible connected holonomy groups. To complete the classification of all connected holonomy groups, one must show that $\operatorname{Sp}(1) \cdot \operatorname{Sp}(r, r)_{W}$ may appear as the holonomy group of a pseudo-quaternionic-Kählerian manifold. 


\section{Proof of Theorem 1}

Let $(M, g)$ be a pseudo-quaternionic-Kählerian manifold of non-zero scalar curvature and of signature $(4 r, 4 s)$. Let $m=r+s$. Obviously, it is enough to prove the theorem in terms of the holonomy algebra $\mathfrak{g} \subset \mathfrak{s p}(1) \oplus \mathfrak{s p}(r, s)$ of $(M, g)$. In 1 it is proved that $\mathfrak{g}$ contains $\mathfrak{s p}(1)$ and it does not preserve any proper non-degenerate subspace of $\mathbb{R}^{4 r, 4 s}$.

For any subalgebra $\mathfrak{g} \subset \mathfrak{s p}(1) \oplus \mathfrak{s p}(r, s)$ denote by $\mathcal{R}(\mathfrak{g})$ the space of algebraic curvature tensors of type $\mathfrak{g}$, i.e. the space of linear maps from $\wedge^{2} \mathbb{R}^{4 r, 4 s}$ to $\mathfrak{g}$ satisfying the first Bianchi identity

$$
R(X, Y) Z+R(Y, Z) X+R(Z, X) Y=0
$$

for all $X, Y, Z \in \mathbb{R}^{4 r, 4 s}$. It is well-known that any $R \in \mathcal{R}(\mathfrak{g})$ satisfies

$$
\eta(R(X, Y) Z, U)=\eta(R(Z, U) X, Y)
$$

for all $X, Y, Z, U \in \mathbb{R}^{4 r, 4 s}$. For example, if $(M, g)$ is a pseudo-quaternionic-Kählerian manifold, $x \in M$, and $\mathfrak{g}$ is the holonomy algebra of $(M, g)$ at the point $x$, then identifying $T_{x} M$ with $\mathbb{R}^{4 r, 4 s}$, we get that the value $R_{x}$ of the curvature tensor $R$ of $(M, g)$ at the point $x$ belongs to $\mathcal{R}(\mathfrak{g})$. From the Ambrose-Singer Theorem 3 it follows that if $\mathfrak{g} \subset \mathfrak{s p}(1) \oplus \mathfrak{s p}(r, s)$ is the holonomy algebra of a pseudo-quaternionic-Kählerian manifold, then it is a Berger algebra, i.e. $\mathfrak{g}$ is spanned by the images of the algebraic curvature tensors $R \in \mathcal{R}(\mathfrak{g})$. We will prove the theorem assuming that $\mathfrak{g} \subset \mathfrak{s p}(1) \oplus \mathfrak{s p}(r, s)$ is a Berger algebra.

Suppose that $\mathfrak{g}$ preserves a degenerate subspace $W \subset \mathbb{R}^{4 r, 4 s}$. Then $\mathfrak{g}$ preserves the isotropic subspace $W \cap W^{\perp}$, i.e. we may assume that $W$ is isotropic. Since $\mathfrak{g}$ contains $\mathfrak{s p}(1), W$ is a quaternionic subspace of $\mathbb{H}^{r, s}$. Let $\operatorname{dim}_{\mathbb{H}} W=t$. Then $W \subset W^{\perp}$ and $\operatorname{dim}_{\mathbb{H}} W^{\perp}=m-t>t$. Let $E$ be a quaternionic subspace of $W^{\perp}$ complementary to $W$, then the restriction of $g$ to $E$ is non-degenerate, let $\left(r_{0}, s_{0}\right)$ be its signature. We have $W \subset E^{\perp}$. Let $W_{1} \subset E^{\perp}$ be any isotropic subspace complementary to $W$. Clearly, $\operatorname{dim}_{\mathbb{H}} W_{1}=\operatorname{dim}_{\mathbb{H}} W$. Let $p_{1}, \ldots, p_{t}, e_{1}, \ldots, e_{r_{0}+s_{0}}, q_{1}, \ldots, q_{t}$ be a basis of $\mathbb{H}^{r, s}$ such that $p_{1}, \ldots, p_{t} \in W, e_{1}, \ldots, e_{r_{0}+s_{0}} \in E, q_{1}, \ldots, q_{t} \in W_{1}$, and the only non-zero values of the pseudo-quaternionic-Hermitian form $g$ are $g\left(p_{i}, q_{i}\right)=g\left(q_{i}, p_{i}\right)=1$ (if $1 \leq i \leq t$ ), $g\left(e_{i}, e_{i}\right)=-1$ (if $\left.1 \leq i \leq r_{0}\right), g\left(e_{i}, e_{i}\right)=1$ (if $r_{0}+1 \leq i \leq r_{0}+s_{0}$ ). Then the Lie algebra $\mathfrak{s p}(r, s)$ can be identified with the following matrix Lie algebra:

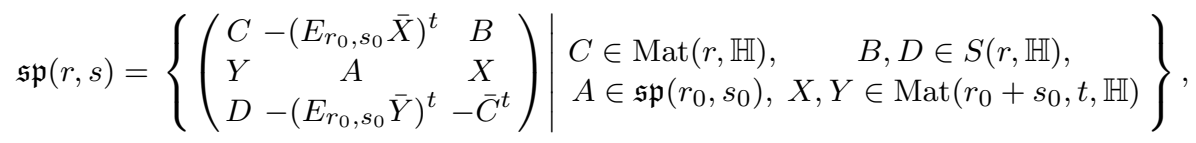

where $\operatorname{Mat}(r, \mathbb{H})$ denotes the space of $r \times r$ quaternionic matrices,

$$
S(r, \mathbb{H})=\left\{B \in \operatorname{Mat}(r, \mathbb{H}) \mid \bar{B}^{t}=-B\right\},
$$

$\operatorname{Mat}\left(r_{0}+s_{0}, t, \mathbb{H}\right)$ denotes the space of $r_{0}+s_{0} \times t$ quaternionic matrices, $E_{r_{0}, s_{0}}=$ $\left(\begin{array}{cc}-E_{r_{0}} & 0 \\ 0 & E_{s_{0}}\end{array}\right)$, and $E_{l}$ denotes the identity $l \times l$ matrix. For the maximal subalgebra $\mathfrak{s p}(r, s)_{W} \subset \mathfrak{s p}(r, s)$ preserving $W$ we get

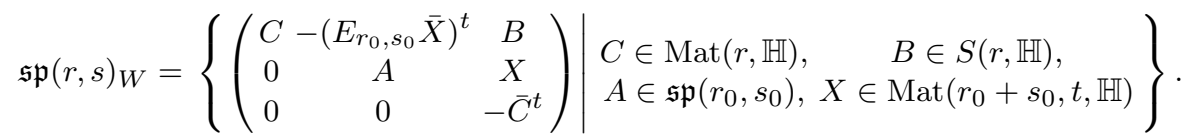


We claim that if $r_{0}+s_{0} \neq 0$, then $\mathcal{R}\left(\mathfrak{s p}(1) \oplus \mathfrak{s p}(r, s)_{W}\right)=\mathcal{R}\left(\mathfrak{s p}(r, s)_{W}\right)$. Let $R \in \mathcal{R}\left(\mathfrak{s p}(1) \oplus \mathfrak{s p}(r, s)_{W}\right)$. From (1) it follows that $R=\nu R_{0}+\mathcal{W}$, where $\nu \in \mathbb{R}, R_{0}$ is given by (2) with $X, Y \in \mathbb{R}^{4 r, 4 s}$, and $\mathcal{W} \in \mathcal{R}(\mathfrak{s p}(r, s))$. Let $p \in W, X \in E$, and $Y, Z \in \mathbb{R}^{4 r, 4 s}$. Using (4), we get

$$
\eta(R(p, X) Y, Z)=\eta(R(Y, Z) p, X)=0,
$$

since $R(Y, Z) p \in W$ and $X \in E \subset W^{\perp}$. Consequently, $R(p, X)=0$. Let $X, Y \in E$, then using the Bianchi identity, we get

$$
R(X, Y) p=-R(Y, p) X-R(p, X) Y=0 .
$$

This shows that $\left.\nu \operatorname{pr}_{W} \circ R_{0}(X, Y)\right|_{W}=-\left.\operatorname{pr}_{W} \circ \mathcal{W}(X, Y)\right|_{W}$. On the other hand, $\operatorname{pr}_{W} \circ$ $\left.\mathcal{W}(X, Y)\right|_{W} \in \mathfrak{g l}(W)=\mathfrak{g l}(r, \mathbb{H})$, whilst $\left.\operatorname{pr}_{W} \circ R_{0}(X, Y)\right|_{W}=\left.\frac{1}{2} \sum_{\alpha=1}^{3} \eta\left(X, I_{\alpha} Y\right) I_{\alpha}\right|_{W}$. Consequently, $\left.\nu \operatorname{pr}_{W} \circ R_{0}(X, Y)\right|_{W}=-\left.\operatorname{pr}_{W} \circ \mathcal{W}(X, Y)\right|_{W}=0$. This means that $\nu \sum_{\alpha=1}^{3} \eta\left(X, I_{\alpha} Y\right) I_{\alpha}=0$. Taking $X=e_{1}, Y=I_{1} e_{1}$, we get $\nu=0$. Thus, $R=$ $\mathcal{W} \in \mathcal{R}\left(\mathfrak{s p}(r, s)_{W}\right)$. This shows that if $r_{0}+s_{0} \neq 0$, then any Berger subalgebra of $\mathfrak{s p}(1) \oplus \mathfrak{s p}(r, s)_{W}$ is contained in $\mathfrak{s p}(r, s)_{W}$.

Let $r_{0}=s_{0}=0$. Then $s=t=r$. From (1) it follows that

$$
\mathcal{R}(\mathfrak{s p}(1) \oplus \mathfrak{s p}(r, r))=\mathbb{R} R_{0} \oplus \mathcal{R}(\mathfrak{s p}(r, r)),
$$

where $R_{0}$ is given by (2) with $X, Y \in \mathbb{R}^{4 r, 4 r}$. Consider the subalgebra

$$
\mathfrak{h}_{0}=\mathfrak{s p}(1) \oplus\left\{\left(\begin{array}{cc}
C & 0 \\
0 & -\bar{C}^{t}
\end{array}\right) \mid C \in \operatorname{Mat}(r, \mathbb{H})\right\} \subset \mathfrak{s p}(1) \oplus \mathfrak{s p}(r, r)_{W} .
$$

This algebra appears as the holonomy algebra of the pseudo-quaternionic-Kählerian symmetric space $\mathrm{SL}(r+1, \mathbb{H}) / \mathrm{S}(\mathrm{GL}(1, \mathbb{H}) \times \mathrm{GL}(r, \mathbb{H}))\left[1\right.$. This shows that $\mathcal{R}\left(\mathfrak{h}_{0}\right)$ contains an element $R_{1}$ such that $\mathfrak{h}_{0}$ annihilates $R_{1} \in \mathcal{R}\left(\mathfrak{h}_{0}\right)$ and the image of $R_{1}$ spans $\mathfrak{h}_{0}$. Let $\mathfrak{g} \subset \mathfrak{h}_{0}$ and $R \in \mathcal{R}(\mathfrak{g})$. Let $X, Y \in W$ and $X_{1} \in W_{1}$. The Bianchi identity (3) implies $R(X, Y) X_{1}=0$ and

$$
R\left(X_{1}, X\right) Y=R\left(X_{1}, Y\right) X
$$

i.e. $R(X, Y)=0$, and for each fixed $X_{1} \in W_{1},\left.R\left(X_{1},\left.\cdot\right|_{W}\right)\right|_{W}$ belongs to the first prolongation $\left(\left.\mathfrak{g}\right|_{W}\right)^{(1)}$ of the subalgebra $\left.\mathfrak{g}\right|_{W} \subset \mathfrak{s p}(1) \oplus \mathfrak{g l}(r, \mathbb{H}) \subset \mathfrak{g l}(4 r, \mathbb{R})$. Similarly, if $X_{1}, Y_{1} \in W_{1}$, then $R\left(X_{1}, Y_{1}\right)=0$; if $X \in W$, then $\left.R\left(X,\left.\cdot\right|_{W_{1}}\right)\right|_{W_{1}} \in\left(\left.\mathfrak{g}\right|_{W_{1}}\right)^{(1)}$. It holds $(\mathfrak{g l}(r, \mathbb{H}))^{(1)}=0\left[\underline{5}\right.$, hence $\mathcal{R}\left(\mathfrak{h}_{0} \cap \mathfrak{s p}(r, r)\right)=0$. From this and (6] it follows that $\mathcal{R}\left(\mathfrak{h}_{0}\right)=\mathbb{R} R_{1}$. This and (6) show that

$$
\mathcal{R}\left(\mathfrak{s p}(1) \oplus \mathfrak{s p}(r, r)_{W}\right)=\mathbb{R} R_{1} \oplus \mathcal{R}\left(\mathfrak{s p}(r, r)_{W}\right) .
$$

Let $\mathfrak{g} \subset \mathfrak{s p}(1) \oplus \mathfrak{s p}(r, r)_{W}$ be a Berger subalgebra such that $\mathfrak{g} \not \subset \mathfrak{s p}(r, r)_{W}$. Then there exists $R \in \mathcal{R}(\mathfrak{g})$ such that $R=\nu R_{1}+\mathcal{W}, \nu \neq 0$ and $\mathcal{W} \in \mathcal{R}\left(\mathfrak{s p}(r, r)_{W}\right)$. Let $p, X \in W$ and $Y, Z \in \mathbb{R}^{4 r, 4 r}$. From (5) applied to $\mathcal{W}$, we obtain $\mathcal{W}(p, X)=0$. From this and the Bianchi identity it follows that $\left.\mathcal{W}\left(X_{1},\left.\cdot\right|_{W}\right)\right|_{W} \in(\mathfrak{g l}(W))^{(1)}=0$ for any $X_{1} \in W_{1}$. Hence, $\left.R\left(X, X_{1}\right)\right|_{W}=\left.\nu R_{1}\left(X, X_{1}\right)\right|_{W}$ for all $X \in W$ and $X_{1} \in W_{1}$. This shows that $\left.\mathfrak{g}\right|_{W}=\left.\mathfrak{h}_{0}\right|_{W}=\left.\mathfrak{s p}(1)\right|_{W} \oplus \mathfrak{g l}(W)$. Suppose that $\mathfrak{g} \neq \mathfrak{h}_{0}$. Then for some $B \in S(r, \mathbb{H})$ the element $\xi=\left(\begin{array}{cc}E_{r} & B \\ 0 & -E_{r}\end{array}\right)$ belongs to $\mathfrak{g}$. If $B \neq 0$, then choosing the basis $p_{1}, \ldots, p_{r}, q_{1}^{\prime}, \ldots, q_{r}^{\prime}$, where $q_{i}^{\prime}=q_{i}-\frac{1}{2} \sum_{j=1}^{r} B_{j i} p_{j}$, we get that $\xi=\left(\begin{array}{cc}E_{r} & 0 \\ 0 & -E_{r}\end{array}\right) \in \mathfrak{g}$. 
Let $\xi_{1}=\left(\begin{array}{cc}C & B \\ 0 & -\bar{C}^{t}\end{array}\right) \in \mathfrak{g}$, where $C \in \operatorname{Mat}(r, \mathbb{H})$ and $B \in S(r, \mathbb{H})$. Then, $\left[\xi, \xi_{1}\right]=$ $\left(\begin{array}{cc}0 & 2 B \\ 0 & 0\end{array}\right) \in \mathfrak{g}$. This shows that $\mathfrak{g}=\mathfrak{h}_{0} \ltimes L$, where $L \subset\left\{\left(\begin{array}{ll}0 & B \\ 0 & 0\end{array}\right) \mid B \in S(r, \mathbb{H})\right\}$. The Lie brackets of elements from $\mathfrak{h}_{0}$ and $L$ are given by the representation of $\mathfrak{g l}(r, \mathbb{H})$ on $S(r, \mathbb{H})$. Since this representation is irreducible $5, \mathfrak{g}=\mathfrak{s p}(1) \oplus \mathfrak{s p}(r, r)_{W}$. It is not hard to see that this algebra is a Berger algebra, hence it is a candidate to be a holonomy algebra.

\section{Proof of Proposition 1}

Let $(M, g)$ be pseudo-quaternionic-Kählerian manifold with the holonomy algebra $\mathfrak{h}_{0}$. Fix a point $x \in M$. Then $T_{x} M$ is identified with $\mathbb{R}^{4 r, 4 r}$. For the covariant derivative of the curvature tensor at the point $x$ we have $\nabla_{X} R_{x} \in \mathcal{R}\left(\mathfrak{h}_{0}\right)$ for any $X \in \mathbb{R}^{4 r, 4 r}$. Let $X, Y \in W$ and $X_{1} \in W_{1}$. From the above, we get $\nabla_{X_{1}} R_{x}(X, Y)=0$. This and the second Bianchi identity imply

$$
\nabla_{X} R_{x}\left(X_{1}, Y\right)=\nabla_{Y} R_{x}\left(X_{1}, X\right)
$$

i.e. $\left.\nabla_{\cdot{ }_{W}} R_{x}\left(X_{1}, \cdot{ }_{W}\right)\right|_{W}$ belongs to the second prolongation of the subalgebra $\mathfrak{s p}(1) \oplus$ $\mathfrak{g l}(r, \mathbb{H}) \subset \mathfrak{g l}(4 r, \mathbb{R})$, which is trivial, since the second prolongation of its complexification $\mathfrak{s l}(2, \mathbb{C}) \oplus \mathfrak{g l}(2 r, \mathbb{C}) \subset \mathfrak{g l}(4 r, \mathbb{C})$ is trivial 11. We get that $\nabla_{X} R_{x}=0$. By the same arguments, $\nabla_{X_{1}} R_{x}=0$. Thus, $\nabla R_{x}=0$ for any $x \in M$, i.e. $\nabla R=0$ and $(M, g)$ is locally symmetric.

\section{Proof of corollaries}

Corollary 1 follows from the above results and from the fact that the only irreducible holonomy group of not locally symmetric pseudo-quaternionic-Kählerian manifolds is $\operatorname{Sp}(1) \cdot \operatorname{Sp}(r, s)[5$. Corollary 3 follows from Corollary 1 and from the results of [1]. To prove Corollary 2 consider the universal covering $(\tilde{M}, \tilde{g})$ of $(M, g)$. Then $(\tilde{M}, \tilde{g})$ is simply connected and complete, hence it is contained in the list of symmetric spaces from 1] given in Introduction. It is known that $M=\tilde{M} / \Gamma$ for some freely acting discrete group $\Gamma$ of isometries of $\tilde{M}$, e.g. 4 .

Acknowledgements I am grateful to D. V. Alekseevsky and Jan Slovák for useful discussions, support and help. The author has been supported by the grant GACR 201/09/H012.

\section{References}

1. D. V. Alekseevsky, V. Cortés, Classification of pseudo-Riemannian symmetric spaces of quaternionic Kähler type. Lie groups and invariant theory, 33-62, Amer. Math. Soc. Transl. Ser. 2, 213, Amer. Math. Soc., Providence, RI, 2005.

2. M. Berger, Sur les groupers d'holonomie des variétés àconnexion affine et des variétés riemanniennes. Bull. Soc. Math. France 83 (1955), 279-330.

3. A. L. Besse, Einstein manifolds, Springer-Verlag, Berlin-Heidelberg-New York, 1987.

4. A. Borel, Semisimple groups and Riemannian symmetric spaces. Texts and Readings in Mathematics, 16. Hindustan Book Agency, New Delhi, 1998. x+136 pp. 
5. R. L. Bryant, Classical, exceptional, and exotic holonomies: a status report. Actes de la Table Ronde de Géométrie Différentielle (Luminy, 1992), 93-165, Semin. Congr., 1, Soc. Math. France, Paris, 1996.

6. A. S. Galaev, T. Leistner, Holonomy groups of Lorentzian manifolds: classification, examples, and applications. Recent developments in pseudo-Riemannian geometry, 53-96, ESI Lect. Math. Phys., Eur. Math. Soc., Zürich, 2008.

7. A. S. Galaev, T. Leistner, Recent developments in pseudo-Riemannian holonomy theory. Cortés, Vicente (ed.), Handbook of pseudo-Riemannian geometry and supersymmetry. Zurich: European Mathematical Society. IRMA Lectures in Mathematics and Theoretical Physics 16, 581-627 (2010).

8. D. Joyce, Riemannian holonomy groups and calibrated geometry. Oxford University Press, 2007.

9. Quaternionic structures in mathematics and physics. Proceedings of the 2nd Meeting held in Rome, September 6-10, 1999. Ed. S. Marchiafava, P. Piccinni and M. Pontecorvo. World Scientific Publishing Co., Inc., River Edge, NJ, 2001. xvi+469 pp.

10. S. M. Salamon, Differential geometry of quaternionic manifolds. Ann. Sci. École Norm. Sup. (4) 19 (1986), no. 1, 31-55.

11. L. J. Schwachhöfer, Connections with irreducible holonomy representations, Adv. Math. 160 (2001), no. 1, 1-80.

12. A. Swann, Hyper-Kähler and quaternionic Kähler geometry. Math. Ann. 289 (1991), no. $3,421-450$. 